\title{
Endoscopic findings of enteropathy-type T-cell
}

\section{lymphoma}

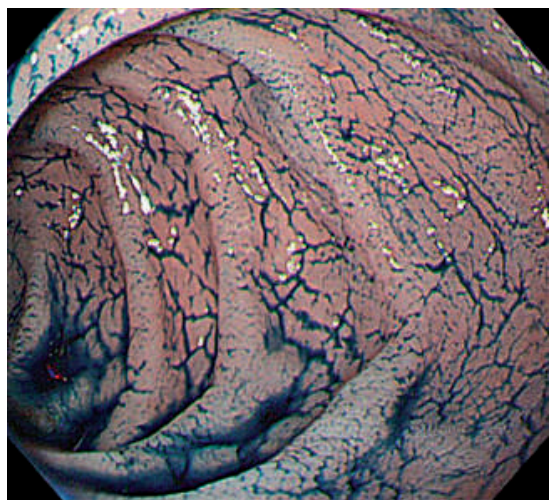

Fig. 1 Patient 1. Esophagogastroduodenoscopic view of the second portion of the duodenum showed edematous mucosa with a nodular or mosaic pattern.
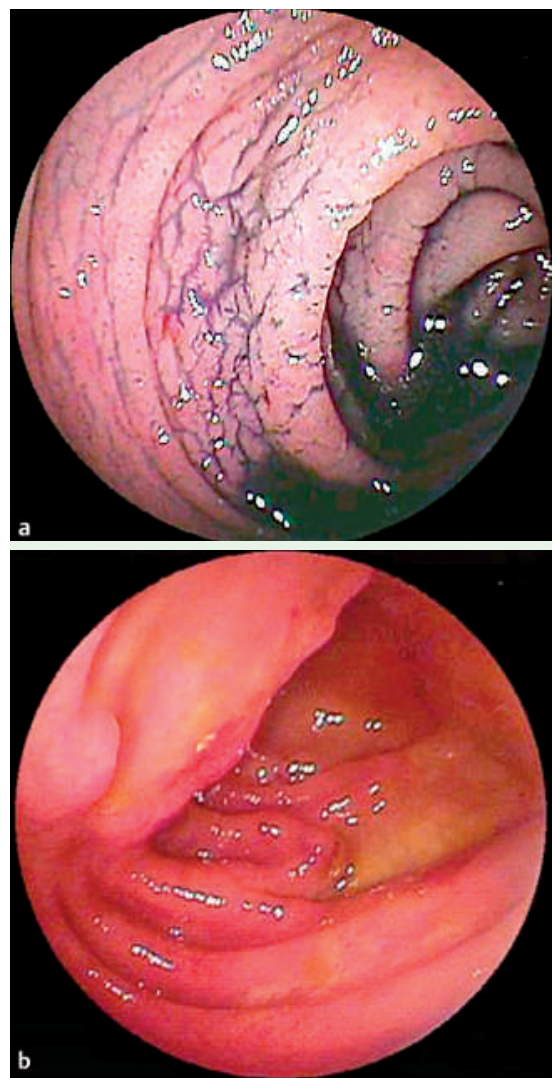

Fig. 2 Patient 1. Antegrade double balloon endoscopic views of the jejunum. a Diffuse thickening of the mucosa with a nodular or mosaic pattern can be seen. $\mathbf{b}$ An ulcerated mass was also observed.
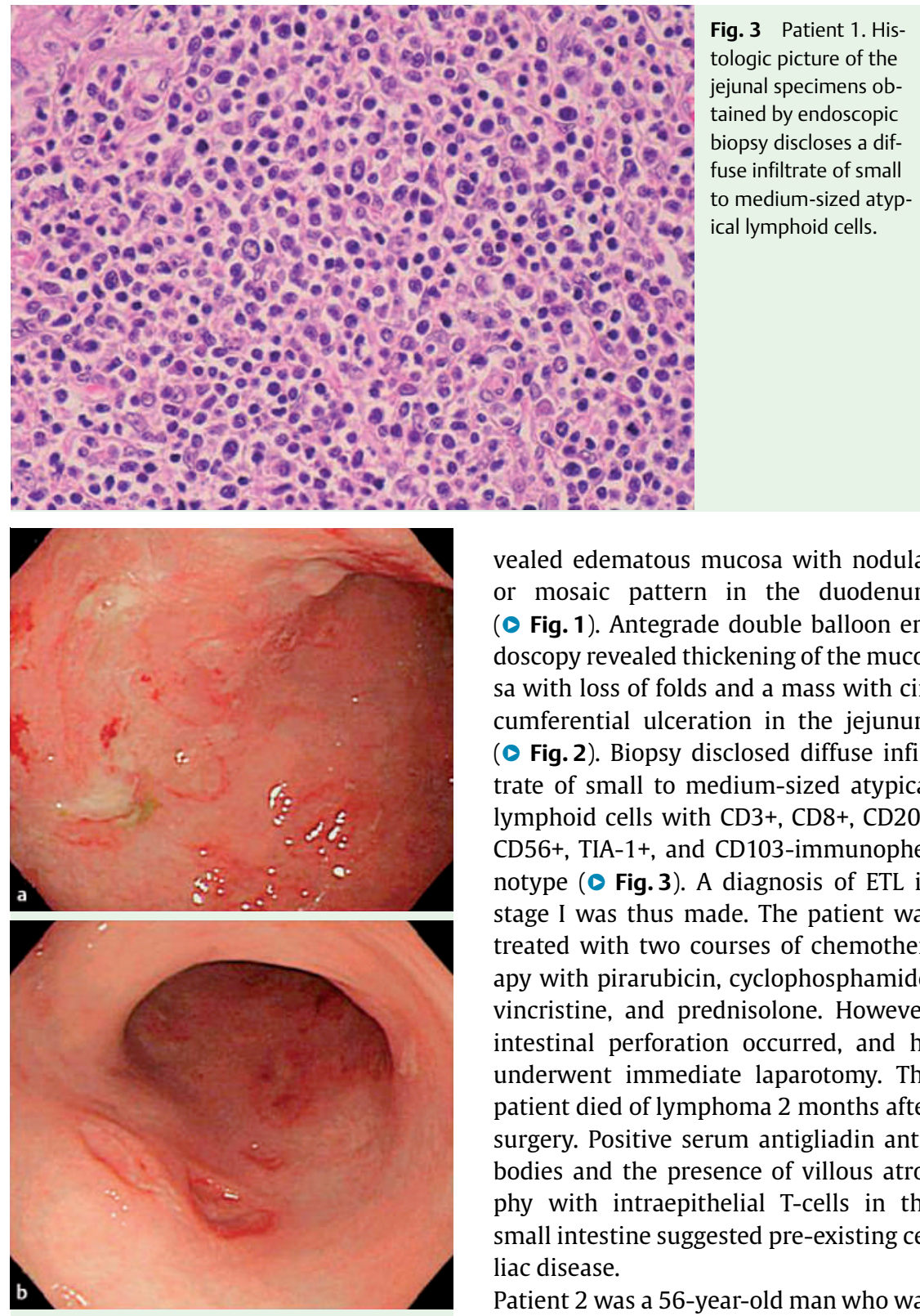

Fig. 4 Patient 2. Colonoscopic views of the sigmoid colon (a) and the rectum (b). Multiple discrete or punched-out ulcers can be seen in the thickened mucosa.

Endoscopic appearance of enteropathytype T-cell lymphoma (ETL) has rarely been described [ $1-4]$. We report detailed endoscopic findings in two Japanese cases of ETL.

Patient 1 was a 77-year-old man who was admitted because of anorexia and diarrhea. Esophagogastroduodenoscopy re- vealed edematous mucosa with nodular or mosaic pattern in the duodenum ( Fig. 1). Antegrade double balloon endoscopy revealed thickening of the mucosa with loss of folds and a mass with circumferential ulceration in the jejunum ( Fig. 2). Biopsy disclosed diffuse infiltrate of small to medium-sized atypical lymphoid cells with CD3+, CD8+, CD20-, CD56+, TIA-1+, and CD103-immunophenotype ( Fig. 3). A diagnosis of ETL in stage I was thus made. The patient was treated with two courses of chemotherapy with pirarubicin, cyclophosphamide, vincristine, and prednisolone. However, intestinal perforation occurred, and he underwent immediate laparotomy. The patient died of lymphoma 2 months after surgery. Positive serum antigliadin antibodies and the presence of villous atrophy with intraepithelial T-cells in the small intestine suggested pre-existing celiac disease.

Patient 2 was a 56-year-old man who was admitted with abdominal pain, diarrhea, and body weight loss (7kg). Sigmoidoscopy revealed multiple discrete ulcers in the edematous sigmoid colon and the rectum ( Fig. 4). After barium meal study, intestinal perforation occurred. During the emergent laparotomy, partial resection of the small intestine was carried out. Histologic examination of the resected intestine suggested a diagnosis of ETL. The patient was treated with four courses of chemotherapy with cyclophosphamide, doxorubicin, vincristine, and predniso- 
lone. However, he died of lymphoma 6 months after the surgery.

Our experiences suggest diffuse mucosal thickening and edema with ulcerations in the intestine to be characteristic of ETL. In addition, double balloon endoscopy showed a nodular or mosaic mucosal pattern in the small intestine, which may be specific to the disease.

Endoscopy_UCTN_Code_CCL_1AC_2AC

S. Yanai ${ }^{1}$, T. Matsumoto ${ }^{1}$, S. Nakamura ${ }^{1}$, K. Fujisawa ${ }^{1}$, T. Ueki ${ }^{2}$, M. Hirahashi ${ }^{3}$,

T. $\mathbf{Y a o}^{3}, \mathbf{M}$. lida ${ }^{1}$

1 Department of Medicine and Clinical Science, Graduate School of Medical Sciences, Kyushu University, Fukuoka, Japan

2 Department of Surgery and Clinical Oncology, Graduate School of Medical Sciences, Kyushu University, Fukuoka, Japan

3 Department of Anatomic Pathology, Graduate School of Medical Sciences, Kyushu University, Fukuoka, Japan

\section{References}

1 Joyce AM, Burns DL, Marcello PW et al. Capsule endoscopy findings in celiac disease associated enteropathy-type intestinal Tcell lymphoma. Endoscopy 2005; 37: $594-$ 596

2 Daum S, Wahnschaffe U, Glasenapp R et al. Capsule endoscopy in refractory celiac disease. Endoscopy 2007; 39: 455-458

3 Hadithi M, Al-toma A, Oudejans J et al. The value of double-balloon enteroscopy in patients with refractory celiac disease. Am J Gastroenterol 2007; 102: 987-996

4 Hadithi M, Akol H, Al-toma A et al. Indigo carmine chromoendoscopic appearances of enteropathy-associated T-cell lymphoma during double-balloon endoscopy in a patient with celiac disease. Endoscopy 2007; [Epub ahead of print]
Bibliography

DOI $10.1055 / s-2007-967063$

Endoscopy 2007; 39: E339-E340

(c) Georg Thieme Verlag KG Stuttgart · New York . ISSN 0013-726X

Corresponding author

\section{S. Yanai, MD}

Department of Medicine and Clinical Science Graduate School of Medical Sciences Kyushu University

Maidashi 3-1-1

Higashi-ku

Fukuoka 812-8582

Japan

Fax: +81-92-6425273

syanai@intmed2.med.kyushu-u.ac.jp 